\title{
A COMPLICATION OF INTRACAPSULAR CATARACT EXTRACTION*
}

BY

\author{
F. A. Williamson-Noble
}

LONDON

IT occasionally happens during this operation, especially in relatively young patients in whom the zonule is tough, that the capsule ruptures. In these circumstances a portion of anterior capsule is removed in the forceps, leaving the rest of the lens behind. Attempts to express the lens, as in the extra-capsular operation, may be unsuccessful owing to the softness of the eyeball, which has been deliberately produced by pre-operative, retro-ocular injection. This

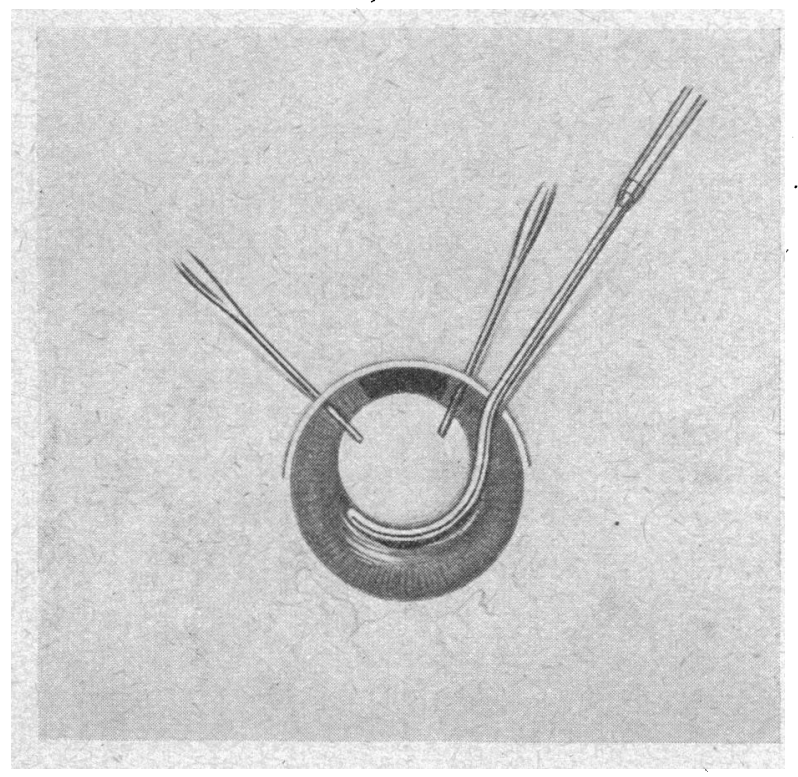

condition is of inestimable value in intracapsular extractions, because it obviates the danger of escape of vitreous, but it may prove a serious obstacle to expression of the lens,' when some of the anterior capsule has been removed. What usually happens is that the lens can be expressed as far as the lips of the wound, but cannot be made to leave the eye. Careful manipulation with a vectis will sometimes effect this, but the use of this instrument increases the risk of intraocular infection and of vitreous loss.

\footnotetext{
- Received for publication, January 11, 1948.
} 
It occurred to the writer once, when faced with this dilemma, to pass two cystitomes into the lens, broadside on, if necessary, steadying the latter during the procedure by gentle pressure with a squint hook held by an assistant (vide figure). The cataract, which was not a soft one, was then easily lifted out of the eye, and no complications ensued. This manoeuvre has been repeated in two other cases with successful results, and is simpler and probably safer than the use of the vectis.

\section{AN ATTEMPT TO TREAT A PERFORATED EYE WITH SUBCONJUNCTIVAL PENICILLIN*}

BY

FRANK R. NEUBERT

GUERNSEY

History. September 17, 1947. Perforation of cornea by piece of wire which patient was cutting.

Examination. The eye was seen about three hours after injury. The cornea had a $3 \mathrm{~mm}$. perforation at 1 o'clock halfway between the pole and limbus. Most of the aqueous was lost. There was no iris prolapse. A thin film of whitish exudate covered the pupil and the lens could not be seen although injury of it was considered likely.

September 18,1947 . There was a sticky conjunctival discharge. The aqueous appeared slightly cloudy. No pain.

September 19, 1947. The right eye was injected. Smear and culture of L.E: shows a small growth of Gram-positive penicillinsensitive cocci. An attempt was made to save the eye by means of penicillin following the method of Sorsby and Ungar. ("Distribution of penicillin in the eye after subconjunctival injection" by Arnold Sorsby and J. Ungar, Brit. Jl. of Ophthal., September, 1947). Mydricain was injected followed by penicillin 50,000 units in adrenalin and novutox subconjunctivally. A course of intra-muscular penicillin injections 30,000 units was commenced.

September 20,1947. The corneal endothelium was almost opaque. The conjunctiva was very injected and the discharge still present. Subconjunctival injection of penicillin was repeated twice.

September 21,1947 . The conjunctival injection was increased and the cornea was quite dull. In view of the apparent deterioration the subconjunctival injections were discontinued. The intramuscular injections were continued.

\footnotetext{
* Received for publication, December 12, 1947.
} 